OPEN ACCESS

Edited by:

Hesham Elhalawani,

Brigham and Women's Hospital and Harvard Medical School, United States

Reviewed by:

Humaid Alshamsi, University of Sharjah, United Arab Emirates Subha Perni,

Brigham and Women's Hospital and Harvard Medical School, United States

${ }^{*}$ Correspondence: Mei Guan

guanmei71@126.com Haitao Zhao

zhaoht@pumch.cn

${ }^{t}$ These authors have contributed equally to this work and share the first authorship

Specialty section: This article was submitted to Gastrointestinal Cancers: Hepato

Pancreatic Biliary Cancers, a section of the journal Frontiers in Oncology

Received: 29 September 2021 Accepted: 02 February 2022 Published: 03 March 2022

Citation:

Wang $Y$, Yang $X$, Wang $D$, Yang $X$, Wang $Y$, Long J, Zhou J, Lu Z, Mao $Y$, Sang $X$, Guan $M$ and Zhao H (2022) Lenvatinib Beyond First-Line Therapy in Patients With Advanced Biliary Tract Carcinoma.

Front. Oncol. 12:785535. doi: 10.3389/fonc.2022.785535

\section{Lenvatinib Beyond First-Line Therapy in Patients With Advanced Biliary Tract Carcinoma}

\author{
Yunchao Wang ${ }^{1 \dagger}$, Xiaobo Yang ${ }^{1 \dagger}$, Dongxu Wang ${ }^{2 \dagger}$, Xu Yang ${ }^{1}$, Yanyu Wang ${ }^{1}$, \\ Junyu Long ${ }^{1}$, Jinxue Zhou ${ }^{3}$, Zhenhui Lu ${ }^{4}$, Yilei Mao ${ }^{1}$, Xinting Sang ${ }^{1}$, Mei Guan ${ }^{5 *}$ \\ and Haitao Zhao $^{1 *}$
}

\begin{abstract}
${ }^{1}$ Department of Liver Surgery, Peking Union Medical College Hospital, Chinese Academy of Medical Sciences and Peking Union Medical College, Beijing, China, 2 Department of Hepatobiliary Surgery, General Surgery, Qilu Hospital, Cheeloo College of Medicine, Shandong University, Jinan, China, ${ }^{3}$ Department of Hepatopancreatobiliary Surgery, The Affiliated Tumor Hospital of Zhengzhou University, Zhengzhou, China, ${ }^{4}$ Hepatobiliary and Pancreatic Surgery, Shenzhen Qianhai Shekou Free Trade Zone Hospital, Shenzhen, China, ${ }^{5}$ Department of Oncology, Peking Union Medical College Hospital, Chinese Academy of Medical Sciences, and Peking Union Medical College, Beijing, China
\end{abstract}

Introduction: Lenvatinib, a multiple receptor tyrosine kinase inhibitors that target vascular endothelial growth factor receptors and fibroblast growth factor receptors, recently demonstrated a treatment effect in various tumors. This study evaluated the efficacy and safety of lenvatinib for patients with biliary tract cancers (BTCs) who had received $\geq 1$ line of prior systemic anti-BTC therapy.

Methods: This open-label, single-arm study included adult ( $\geq 18$ years) patients with histologically confirmed BTC. Efficacy and safety were evaluated based on the Response Evaluation Criteria in Solid Tumors RECIST Version 1.1 (RECIST 1.1) and the National Cancer Institute Common Terminology Criteria for Adverse Events (CTCAE version 4.0). Changes in tumor biomarkers throughout the treatment period were recorded.

Results: 41 patients received lenvatinib treatment. The ORR was 12\% (95\% Cl: $1.7-$ 22.7), with a median PFS of 3.8 months (95\% Cl: 1.3-6.3) and an OS of 11.4 months (95\% Cl: 6.6-16.2). Thirty-nine (95.1\%) patients experienced $\geq 1$ treatment-related adverse event. Decreasing carbohydrate antigen 19-9 (CA19-9) level predicted tumor size reduction in intrahepatic cholangiocarcinoma with a sensitivity of $77.7 \%$ and a specificity of $73.9 \%$.

Conclusions: Lenvatinib which was individualized based on the patient's weight has promising clinical activity against advanced BTC and had an acceptable safety profile. Additionally, serum biomarkers and gene sequencing may hold the potential to guide our treatment.

Keywords: lenvatinib, advanced biliary tract carcinoma, efficacy, biomarker, tyrosine kinase inhibitor 


\section{INTRODUCTION}

Biliary tract cancers (BTCs), encompassing intrahepatic cholangiocarcinoma (ICC), extrahepatic cholangiocarcinoma (ECC), and gallbladder cancer (GBC), are aggressive neoplasms with a dismal prognosis (1). The global incidence of BTCs has increased in recent years, in large part due to an increase in the incidence of ICC (2). Surgical resection is regarded as a curative treatment option for patients with earlystage BTC. However, most patients present with unresectable or metastatic disease at diagnosis. Presently, gemcitabine combined with cisplatin (GC) chemotherapy is the standard first-line treatment for advanced BTCs (3-5), and the incidence of adverse reactions accounted for $70 \%$. The ABC-06 study evaluated the benefit derived from FOLFOX (folinic acid, fluorouracil, and oxaliplatin) chemotherapy in patients with advanced BTC and the median overall survival (OS) was improved significantly compared to active symptom control (ASC) (6.2 months vs 5.3 months). However, the absolute benefit was not large, and the median OS was only prolonged by 0.9 months (6). At the same time, the incidence of the grade 3 5 AEs associated with FOLFOX chemotherapy account for $69 \%$. For patients who progress on or after the above therapies, the subsequent treatment options are limited.

Multiple molecular pathways, including those mediated by vascular endothelial growth factor (VEGF) and fibroblast growth factor receptor (FGFR), are crucial in the carcinogenesis and development of BTC. In particular, overexpression of VEGF occurs in $42 \%$ to $76 \%$ of patients with advanced unresectable BTC and is associated with a poor prognosis (7). Multiple small studies of treatments targeting the VEGF pathway (sorafenib, cabozantinib, bevacizumab, sunitinib, or axitinib) have been conducted in BTC and showed modest efficacy (8-11). Furthermore, FGFR plays an important role in regulating cell proliferation, migration, invasion, and angiogenesis in BTC. Previous studies have found FGFR fusions in patients with BTC and shown that the presence of these alterations predicts a more favorable prognosis (7). Several clinical trials of drugs that target FGFR-fusions have been conducted, including infigratinib, pemigatinib, and derazantinib (12).

Lenvatinib is a small-molecule tyrosine kinase inhibitor of VEGFR1-3, FGFR1-4, platelet-derived growth factor receptor a (PDGFRa), stem cell factor receptor (KIT), and rearranged during transfection (RET). Lenvatinib promotes tumor shrinkage in hepatocellular carcinoma (13), endometrial carcinoma $(14,15)$, and renal cell cancer (16). In a previous study, we found lenvatinib plus pembrolizumab was a potentially effective and tolerable non-first-line treatment for refractory BTC, with an objective response rate (ORR) of $25 \%$, a clinical benefit rate (CBR) of $40.5 \%$, a median progression-free survival (PFS) of 4.9 months, and a median OS of 11.0 months (17). As lenvatinib can inhibit multiple kinases that are implicated in BTC carcinogenesis, and due to the positive effects observed when lenvatinib is used as a combination therapy, we sought to test lenvatinib monotherapy, as this may provide a better safety profile while remaining efficacious. In the present study, we investigated the efficacy and safety of lenvatinib monotherapy in patients with advanced BTC.

\section{MATERIALS AND METHODS}

\section{Study Design}

This was an open-label, single-arm study designed to assess the efficacy and safety of lenvatinib as a non-first-line treatment in patients with advanced BTC. Clinical data from patients with advanced BTC treated with lenvatinib in second-line or beyond were collected from December 2017 until July 2019. All patients had been diagnosed with unresectable BTC and were routinely attending for a treatment consultation. The study protocol was compliant with the Declaration of Helsinki and was approved by the Institutional Review Board and Ethics Committee at Peking Union Medical College Hospital. All patients provided written, informed consent before enrolment. The study protocol was prospectively registered at ClinicalTrials.gov (NCT04656249).

\section{Patients}

Eligible patients were aged $\geq 18$ years with histologically confirmed ECC, ICC, or GBC and had previously received at least one systemic anti-BTC therapy. Other eligibility criteria included at least one measurable or evaluable tumor lesion according to Response Evaluation Criteria in Solid Tumors version 1.1 (RECIST 1.1) and an Eastern Cooperative Oncology Group performance status of $0-2$. Participants who received adjuvant chemotherapy were eligible if this therapy was completed and no recurrence was observed for 6 months after completion of the therapy. Key exclusion criteria included intolerance to lenvatinib, the life expectancy of $\leq 3$ months, moderate or severe ascites or ascites requiring drainage, proteinuria of $\geq 2+$ on dipstick testing, gastrointestinal malabsorption, or any other condition that the investigator determined may affect the absorption of study drug, cardiovascular disease, HIV infection, and gastrointestinal bleeding.

\section{Treatment}

Patients with a bodyweight $<60 \mathrm{~kg}$ received $8 \mathrm{mg}$ of lenvatinib and those with a bodyweight $\geq 60 \mathrm{~kg}$ received $12 \mathrm{mg}$ orally once a day in 28-day cycles until disease progression or intolerable toxicity occurred. Dose interruption and reduction of lenvatinib (to $10 \mathrm{mg} \mathrm{QD}$ ) were permitted for drug-related adverse events. Lenvatinib could be interrupted and even discontinued with the occurrence of unacceptable or serious adverse events (AEs) or due to disease progression.

\section{Assessments}

We evaluated tumor size using enhanced computed tomography (CT), magnetic resonance imaging (MRI), or other available imaging examinations according to RECIST 1.1 at 4-8 weeks after the patient-initiated lenvatinib at the stipulated dose. Imaging examination was conducted by a specialist to assess tumor size changes and each patient was categorized as either complete response (CR), partial response (PR), stable disease 
$(\mathrm{SD})$, or progressive disease $(\mathrm{PD})$. Disease control rate (DCR; $\mathrm{CR}+\mathrm{PR}+\mathrm{SD})$ and $\mathrm{ORR}(\mathrm{CR}+\mathrm{PR})$ were also calculated.

The therapeutic efficacy included ORR, PFS, OS, DCR, and safety. Treatment-related AEs were categorized and graded according to the National Cancer Institute Common Terminology Criteria for Adverse Events, version 4.0 (CTCAE 4.0). The highest grade for each $\mathrm{AE}$ experienced by each patient during the observation period was recorded. Treatment of AE symptoms was incorporated into treatment regimens for patients experiencing grade 1 or 2 AEs. IF grade 3 AEs occurred, the treatment dose was reduced or treatment was temporarily interrupted until symptoms were reduced to grade 1 or 2 . If grade 4 AEs occurred, the administration of lenvatinib was discontinued.

\section{Tumor Serum Biomarker and Gene Sequence Data Analysis}

Serum concentrations of carbohydrate antigen 19-9 (CA19-9) were recorded every 6-8 weeks from lenvatinib initiation until treatment discontinuation or completion of the study period. We assessed the ability of biomarker values before and after treatment to predict the direction and amount of change in tumor size by receiver operating characteristic (ROC) curve analysis. In addition, patients with BTC were divided into the following groups: partial response (PR), diminished tumor size that did not reach the partial response standard (SS), stable disease without any significant tumor size change (ST), and progressive disease (PD). Gene mutation information was collected from the patients by performing gene sequencing of tumor tissue. For each group, different information representing the gene mutation was calculated.

\section{Statistical Analysis}

PFS and OS were calculated from the initiation of lenvatinib until disease progression or BTC-related death. The clinical baseline data of patients including continuous variables and categorical variables were summarized as averages with corresponding standard deviations or simple numbers and percentages according to their types. The Kaplan-Meier method was used to generate PFS and OS curves. The sensitivity and specificity of diagnostics were calculated to assess their predictive capabilities for tumor changes using CA19-9 values. Gene mutation frequencies were used to construct a gene mutation map of patients with different therapeutic outcomes. SPSS software system (vision 22.0, SPSS, Inc., Chicago, IL), and GraphPad Software (vision 8.0, GraphPad Software, San Diego, California) were used for statistical analysis.

\section{RESULTS}

\section{Patient Demographics and Baseline Characteristics}

From December 2017 until July 2019, a total of 46 patients were enrolled. Of these patients, five were excluded: 4 patients without measurable target lesions, 1 patient withdrew consent before treatment (Figure 1). The mean age was $61 \pm 12$ years, and $61 \%$ were male. In total, 34 patients $(82.9 \%)$ had ICC, three $(7.3 \%)$ had ECC, and four (9.8\%) had GBC. There were six patients with a hepatitis B infection. Twenty-three patients had received systemic chemotherapy (primarily gemcitabine with either cisplatin or oxaliplatin). Twenty-one patients (51.2\%) had received more than one line of systemic treatment before initiating lenvatinib. Five patients $(12.2 \%)$ had also received radiation therapy, and 11 patients $(26.8 \%)$ had undergone transhepatic arterial chemoembolization. Seven patients (17.1\%) had received radiofrequency ablation. Baseline characteristics of the patients included in this study are shown in Table 1.

\section{Efficacy and Safety}

Among 41 patients evaluable for tumor response, none achieved a CR, five achieved a PR, 27 had SD, and nine had PD (Table 2). The ORR was $12.2 \%$ (5/41; 95\% CI: $1.7-22.7)$ and the DCR was 78.0\% (32/41; 95\% CI: 64.8-91.3). The median PFS was 3.8 months (95\% CI: 1.3-6.3) and the OS was 11.4 months (95\% CI: 6.6-16.2) (Figure 2). A total of 39 patients (95.1\%) experienced $\geq$ 1 treatment-related $\mathrm{AE}$, the most common of which were fatigue $(46.3 \%, \mathrm{n}=19)$ followed by hypertension (43.9\%, $\mathrm{n}=18$ ). Eighteen patients (19.5\%) experienced grade 3-4 AEs, of which diarrhea and hypertension were the most common (both $\mathrm{n}=2$, 4.9\%). A detailed list of AEs and their associated frequencies are shown in Table 3 and Figure 3.

\section{Treatment Response Based on CA19-9 and Gene Mutations}

Of the 41 patients, $48.8 \%(n=20)$ showed decreased CA19-9 concentrations after initiation of lenvatinib. Prediction of DCR using CA19-9 had a sensitivity of 59\%, but specificity was substantially higher at $88.8 \%$ (Table 2 ).

Gene mutation data from 28 patients in the BTC group was analyzed. These patients were divided into two groups by tumor response: a group with reduced tumor size $(n=15)$ and a group characterized by stable or enlarged tumors (the 'non-response' group, $\mathrm{n}=13$ ). The majority of BTC mutations involved TP53 $(\mathrm{n}=10), \operatorname{KRAS}(\mathrm{n}=7)$, and DNMT3A $(\mathrm{n}=5)$. Alterations in $I D H 1, F G F 1$, $R B 1$, and $B A P 1$ appeared in patients with tumor size reduction, but not in those with non-responding tumors. On the other hand, alterations in NOTCH1, CDKN2A, ARID2, EGFR, SETD2, PMS2, NTRK1, CCND1, and ATM were found in patients with non-responding tumors, but not in those with tumor size reductions (Figure 4).

\section{DISCUSSION}

The purpose of this study was to evaluate the safety and efficacy of lenvatinib as beyond first-line treatment in patients with unresectable BTC. Lenvatinib showed antitumor activity, with an ORR of $12.2 \%$ (95\% CI: 1.7-22.7), a DCR of $78.0 \%$ (95\% CI: $64.8 \%-91.3 \%$ ), and a median OS of 11.4 months (95\% CI: 6.616.2). A majority of the enrolled patients $(95.1 \%, 39 / 41)$ experienced $\geq 1$ treatment-related $\mathrm{AE}$, and the incidence of 


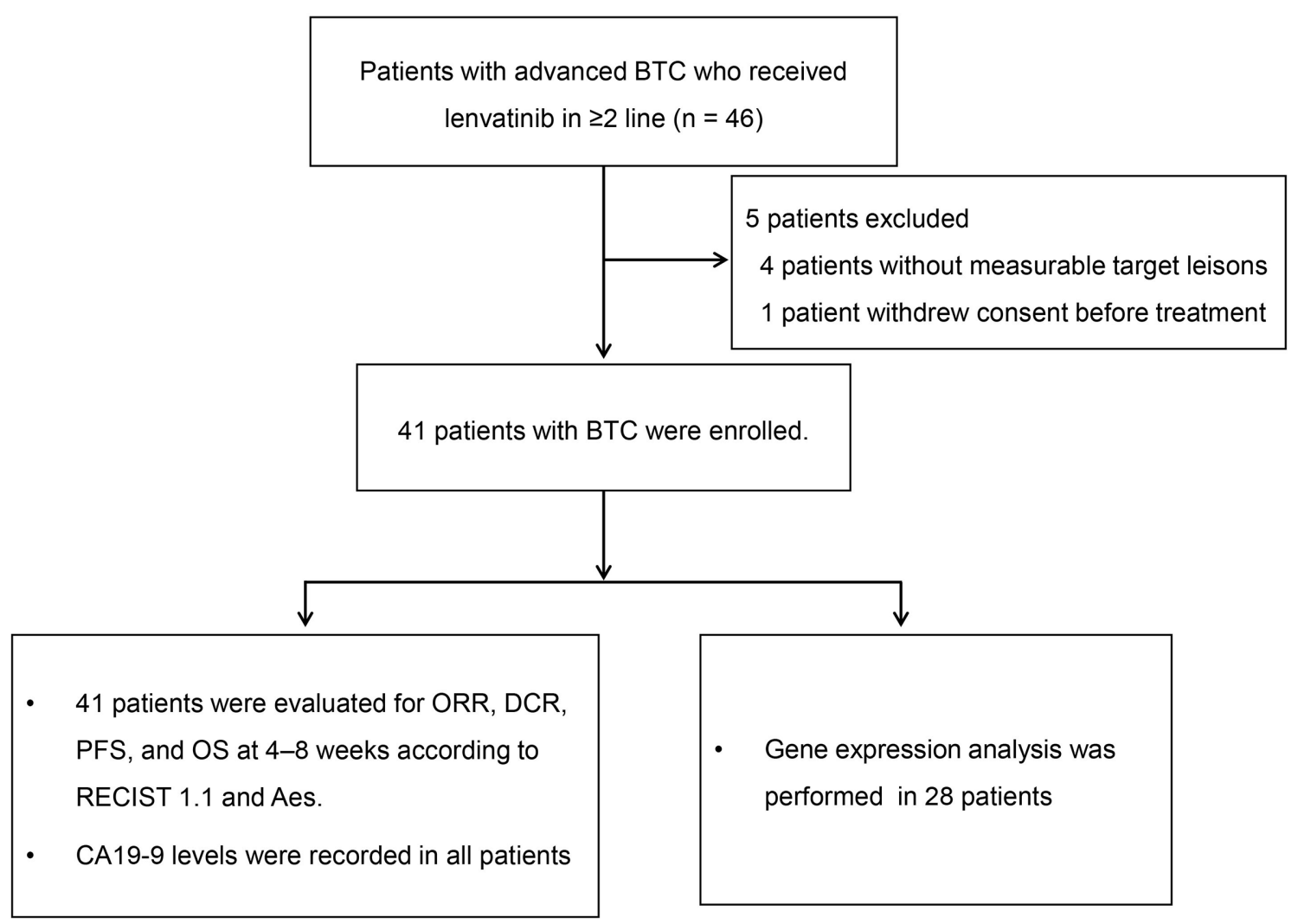

FIGURE 1 | Study flow diagram. BTC, biliary tract cancer; CA19-9, carbohydrate antigen 19-9; DCR, disease control rate; ORR, objective response rate; OS, overall survival; PFS, progression-free survival; RECIST 1.1, Response Evaluation Criteria in Solid Tumors version 1.1.

grade 3-4 AEs was 19.5\% $(\mathrm{n}=8)$. The most common AE was fatigue $41.3 \%(n=19)$, followed by hypertension $39.1 \%(n=18)$. The sensitivity and specificity of CA19-9 serum concentration before and after treatment for predicting tumor shrinkage were $77.7 \%$ and $73.9 \%$, respectively, while the sensitivity and specificity for predicting DCR were $59.3 \%$ and $88.8 \%$. The most commonly mutated genes in patients with tumor shrinkage were DNMT3A, TP53, and KRAS.

Surgical resection is the best option for patients with localized disease. However, most BTC patients are at an advanced stage when diagnosed (5). The ABC-02 trial established the combination of GC as a first-line treatment in BTC patients, with GC showing a better OS than gemcitabine alone (median OS: 11.7 months vs 8.1 months). Only around $26 \%$ of patients respond to this chemotherapy regimen, and the efficacy is limited (3). For patients with disease progression following treatment with cisplatin and gemcitabine, the FOLFOX as a second-line treatment showed a modest survival benefit from the ABC-06 trial (6). The study reported an mOS of 6.2 months, mPFS of 4.0 months in the ASC plus mFOLFOX arm. However, ORR was only $5 \%$ and DCR was $33 \%$. For patients who progress after the above therapies, the subsequent treatment options are limited. Moreover, some patients have difficulty tolerating the many side effects of chemotherapy drugs. Therefore, additional therapies are needed.

Multi-kinase small molecule inhibitors have been investigated the efficacy and safety in patients with biliary tract cancers (BTCs). A phase II study of sorafenib, a kinase inhibitor of VEGFR2/3, PEGFR, and RAF, evaluating the safety and efficacy of sorafenib in patients with unresectable or metastatic gallbladder carcinoma and cholangiocarcinoma was terminated because it did not result in objective responses (ORR:0\%) (18). Regorafenib as a single agent in patients with advanced BTC showed clinical efficacy with an ORR of $11 \%$ and an mPFS of 15.6 weeks (90\% CI: 12.9 24.7 weeks) (19). The activity of anti-VEGF targeted therapies as monotherapy in advanced BTC is not very satisfactory. Recently, infigratinib (BGJ398), a selective FGFR kinase inhibitor, has shown promising results against chemotherapy-refractory CCA harboring FGFR2 fusions (NCT02150967). In that study, the ORR was $14.8 \%$ (18.8\% in those with FGFR2 fusions), the DCR was $75.4 \%$ (83.3\% in those with FGFR2 fusions), an estimated median PFS was 5.8 months (95\% CI: 4.3-7.6) (20). The FDA has approved the infigratinib for patients with locally advanced or metastatic cholangiocarcinoma. Another study showed that ivosidenib (AG-120) targeted IDH1 mutations could improve survival outcomes in participants with histologically confirmed 
TABLE 1 | Patient demographics and baseline characteristics.

\begin{tabular}{|c|c|}
\hline Characteristics & $n=41$ \\
\hline Age, years & $61.10 \pm 12.50$ \\
\hline \multicolumn{2}{|l|}{ Gender, n (\%) } \\
\hline Male & $25(61.0)$ \\
\hline Female & $16(39.0)$ \\
\hline \multicolumn{2}{|l|}{ Tumor subtype, n (\%) } \\
\hline ECC & $3(7.3)$ \\
\hline ICC & $34(82.9)$ \\
\hline GBC & $4(9.8)$ \\
\hline \multicolumn{2}{|l|}{ ECOG performance status, n (\%) } \\
\hline 0 & $12(29.3)$ \\
\hline 1 & $25(61.0)$ \\
\hline 2 & $4(9.8)$ \\
\hline HBV infection & $6(15.6)$ \\
\hline \multicolumn{2}{|l|}{ Differentiated histology, n (\%) } \\
\hline Well & $1(2.4)$ \\
\hline Moderately & $8(19.5)$ \\
\hline Poorly & $3(7.3)$ \\
\hline Moderately-poorly & $7(17.1)$ \\
\hline Unsure & $22(53.7)$ \\
\hline Total bilirubin, mg/dL & $21.33 \pm 22.43$ \\
\hline Albumin, $g / d L$ & $37.77 \pm 8.51$ \\
\hline \multicolumn{2}{|l|}{ Site of Metastases n, (\%) } \\
\hline Intrahepatic & $29(70.7)$ \\
\hline Lymph nodes & 30 (73.2) \\
\hline Bone & $1(2.4)$ \\
\hline Lung & $1(2.4)$ \\
\hline Other & $2(4.9)$ \\
\hline \multicolumn{2}{|l|}{ Extent of disease, $\mathrm{n}(\%)$} \\
\hline Native & $21(51.2)$ \\
\hline Recurrence & $20(48.8)$ \\
\hline \multicolumn{2}{|l|}{ Previous treatment regimens, n (\%) } \\
\hline Radical surgery resection & $19(46.3)$ \\
\hline Systemic chemotherapy & $23(56.1)$ \\
\hline TACE & $11(26.8)$ \\
\hline RFA & $7(17.1)$ \\
\hline Radiotherapy & $5(12.2)$ \\
\hline$\geq 2$ lines of previous systemic treatments, $n(\%)$ & $21(51.3)$ \\
\hline \multicolumn{2}{|l|}{ Initial dose of lenvatinib, n (\%) } \\
\hline $8 \mathrm{mg}$ & $21(51.2)$ \\
\hline$>8 \mathrm{mg}$ & $20(40.8)$ \\
\hline Hepatocirrhosis, n (\%) & $3(7.3)$ \\
\hline Gastric varices and portal hypertension, n (\%) & $3(7.3)$ \\
\hline
\end{tabular}

TABLE 2 | Tumor responses based on RECIST 1.1 and predictive ability of biomarkers.

\begin{tabular}{lc}
\hline Response by investigator review (RECIST 1.1) & $\mathbf{N}=\mathbf{4 1}$ \\
\hline Median overall survival, months (95\% Cl) & $11.4(6.6-16.2)$ \\
Median progression-free survival, months (95\% Cl) & $3.8(1.3-6.3)$ \\
Objective response rate (n, \%), 95\% Cl & $5(12.2), 1.7-22.7$ \\
Complete response (n, \%) & $0(0)$ \\
Partial response (n, \%) & $5(12.2)$ \\
Stable disease (n, \%) & $27(65.9)$ \\
Progressive disease (n, \%) & $9(22.0)$ \\
DCR (n, \%), 95\% Cl & $32(78.0), 64.8-91.3$ \\
Decreased CA19-9 predict DCR status & \\
Sensitivity, \% & 59.0 \\
Specificity, \% & 88.8 \\
\hline
\end{tabular}

CA19-9, carbohydrate antigen 19-9; DCR, disease control rate; RECIST, Response Evaluation Criteria in Solid Tumors.

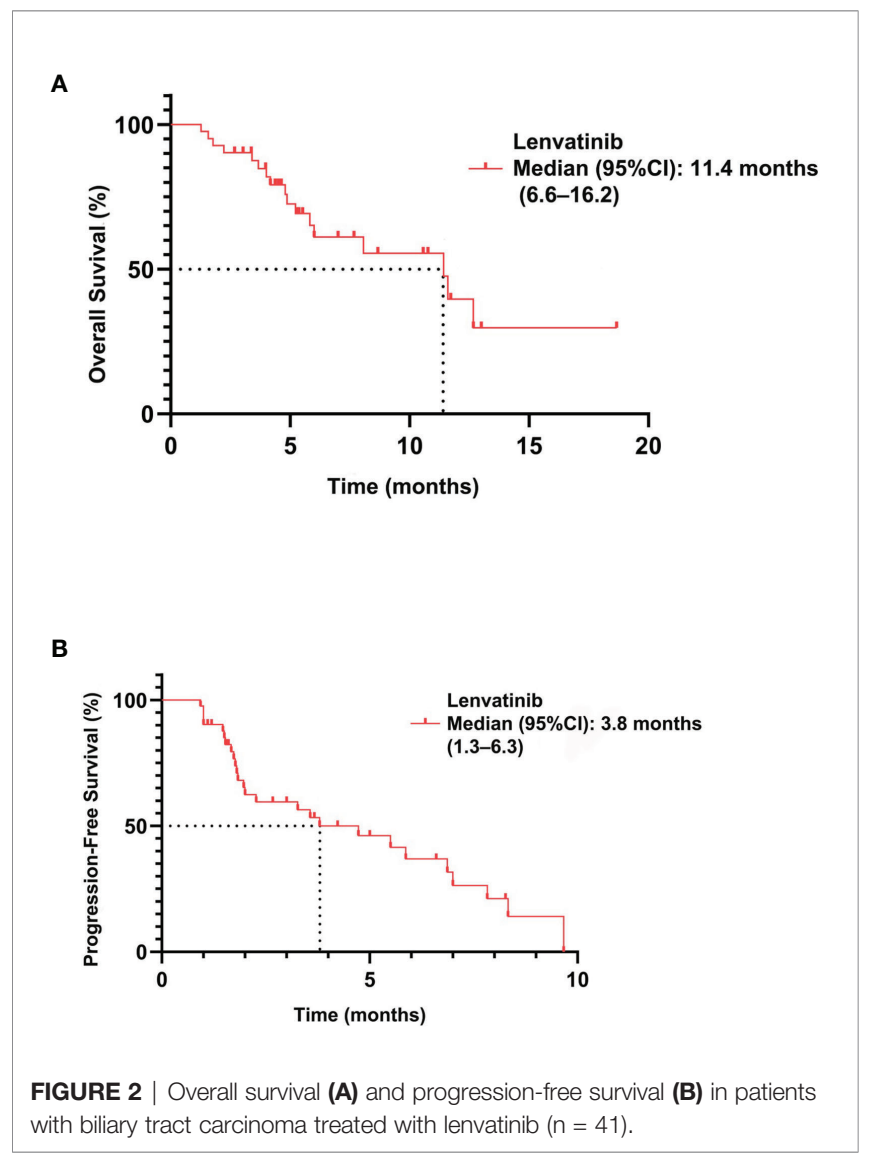

IDH1-positive chemotherapy-refractory cholangiocarcinoma, with a PFS of 2.7 months (IQR 1.6-4.2) (21). Ivosidenib and infigratinib are effective for patients with specific gene mutations. Our study enrolled patients regardless of specific gene mutation.

Lenvatinib not only inhibits VEGFR1-3, FGFR1-4, PDGFRa, KIT, and RET but also demonstrates immunomodulatory activity. Lenvatinib has proven to be effective in many types of

TABLE 3 | Summary of adverse events.

\section{Events, n (\%)}

Biliary tract carcinoma $(\mathrm{N}=41)$

\begin{tabular}{lcc} 
& Any grade & Grade 3-4 \\
\cline { 2 - 3 } Hypertension & $18(43.9)$ & $2(4.9)$ \\
Fatigue & $19(46.3)$ & $0(0)$ \\
Decreased appetite & $12(29.3)$ & $0(0)$ \\
Diarrhoea & $4(9.6)$ & $2(4.9)$ \\
Proteinuria & $3(7.3)$ & $1(2.4)$ \\
Decreased weight & $7(17.1)$ & $0(0)$ \\
Palmar-plantar erythrodysaesthesia & $5(12.2)$ & $1(2.4)$ \\
Nausea & $4(9.6)$ & $0(0)$ \\
Abdominal pain & $4(9.6)$ & $0(0)$ \\
Rash & $5(12.2)$ & $0(0)$ \\
Decreased platelet count & $4(9.6)$ & $1(2.4)$ \\
Vomiting & $3(7.3)$ & $0(0)$ \\
Hypothyroidism & $1(2.4)$ & $0(0)$ \\
Elevated aspartate aminotransferase & $3(7.3)$ & $0(0)$ \\
Increased blood bilirubin & $2(4.9)$ & $1(2.4)$ \\
Constipation & $1(2.4)$ & $0(0)$
\end{tabular}




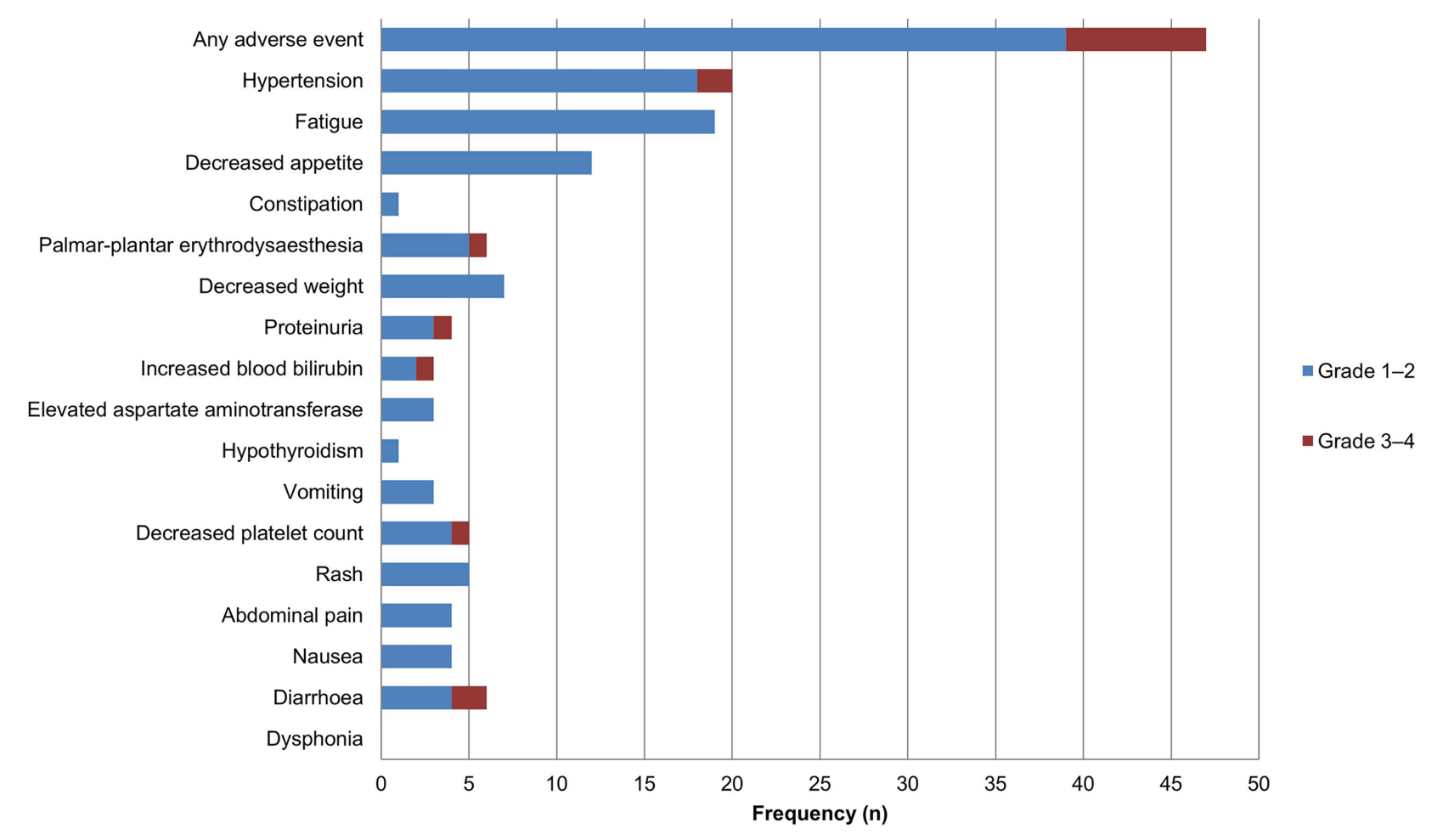

FIGURE 3 | Adverse events during lenvatinib treatment in patients with biliary tract carcinoma $(n=41)$. Grade $1-2$ adverse events are represented in blue, and grade 3-4 adverse events are represented in red.

cancer. However, there have been few studies on the efficacy of lenvatinib in BTC. A phase II study of lenvatinib monotherapy as second-line treatment in 26 patients with unresectable BTC reported an ORR of $11.5 \%$, a median PFS of 3.19 months, and a median OS of 7.35 months (Study 215). The present study showed better results than Study 215, with a median PFS of 3.8 months and an OS of 11.4 months (22). An important factor behind the longer OS and PFS is that the BTC subtypes were represented differently between studies; patients with ICC accounted for only $23.1 \%$ of patients in that study compared to $82.9 \%$ in our study. Moreover, all patients in that study received a higher dose of lenvatinib (24 mg orally once daily), in contrast to our study in which the lenvatinib dosage was individualized based on the patient's weight, to a maximum dose of $12 \mathrm{mg}$ daily.

Evidence has shown that lenvatinib exerted immunomodulatory effects $(23,24)$. We have reported that lenvatinib plus pembrolizumab as a non-first-line treatment for BTC was potentially effective and tolerable, with an ORR of $25 \%$, a CBR of $40.5 \%$, a median PFS of 4.9 months, and a median OS of 11.0 months (17). These results showed that lenvatinib plus anti-PD-1 might have promising antitumor activity in patients with advanced BTC. Dual anti-PD-1 and VEGF/VEGFR blockade may reprogram the tumor microenvironment and enhance the antitumor efficacy of immunotherapy targeting PD1/PDL1 (25).
Regarding safety and tolerability, we found that $95.1 \%$ of patients experienced treatment-related AEs; however, the incidence of grade 3-4 AEs was $19.5 \%$, and there were no grade 5 AEs. Of the grade 3-4 AEs, diarrhea and hypertension were the most common ( $4.9 \%$ each). The most common anygrade $\mathrm{AE}$ was fatigue $(41.3 \%, \mathrm{n}=19)$, followed by hypertension $(39.1 \%, \mathrm{n}=18)$, and these side effects were generally tolerable. In Study $215,80.8 \%$ of patients experienced grade $\geq 3$ treatmentemergent AEs, which is over four times the rate observed in our study (22). In our study, patients with a bodyweight $<60 \mathrm{~kg}$ received $8 \mathrm{mg}$ of lenvatinib daily, and those with a bodyweight $\geq 60 \mathrm{~kg}$ received $12 \mathrm{mg}$. Thus, lenvatinib side effects appear to be closely related to the administered dose, and this may explain the differences between the safety findings of the two studies. Besides, we timely found and treated the AEs of each patient through social platforms. As hypertension, diarrhea, and proteinuria are serious threats to a patient's quality of life, it is important to ensure timely control of AEs and interrupt lenvatinib treatment if necessary.

Tumor biomarkers represent tumor activity under certain circumstances, and clinicians may use changes in tumor biomarkers to assist in understanding and predicting treatment effects. CA19-9 levels play an important role in the diagnosis of BTC. Results from this study suggest that a downward trend 


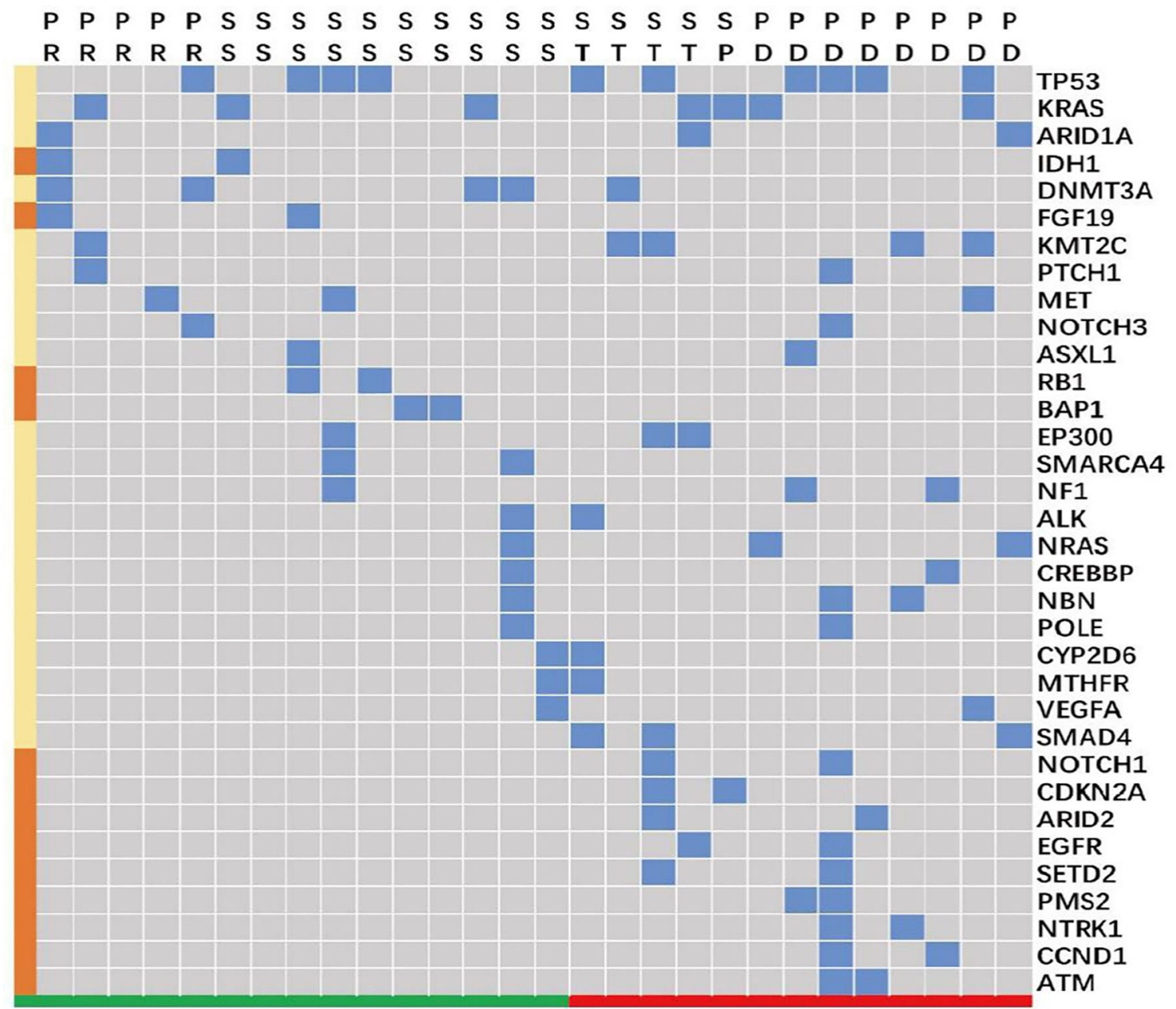

FIGURE 4 | Gene alterations in patients with biliary tract carcinoma $(n=28)$. SS and PR (colored in green along the bottom) clustered as a group characterized by tumor size reduction in response to treatment. ST, SP, and PD (colored in red) clustered as a 'non-response' group, which included patients who did not respond to treatment with tumor size reduction. The blue blocks represent the presence of an alteration in the indicated gene across individual patients, and the brown blocks along the left indicate that the gene alteration was present only either in the tumor size reduction group (IDH1, FGF19, RB1, and BAP1) or in the "unshrinking" group (NOTCH1, CDKN2A, ARID2, EGFR, SETD2, PMS2, NTRK1, CCND1, and ATM). PD, progressive disease; PR, partial response; SS, diminished tumor size that did not reach the partial response standard; ST, a stable disease without any significant tumor size change.

ofCA19-9 levels after initiating lenvatinib is representative of response to treatment. Upon further analysis, we found this response to be highly sensitive and specific for predicting the reduction of tumor volume. Therefore, CA 19-9 may serve as a biomarker in the treatment of BTC, and clinicians may be able to adjust lenvatinib treatment plans in accordance with decreasing serum markers. However, this is an area that requires further validation in future studies.

BTC is a highly heterogeneous malignancy (26). Gene sequencing is necessary to pave the way for new treatment options and personalized medicine. In a study of BTC targets, potential driver mutations were detected in a majority of patients, and $40 \%$ of these mutations could be targeted with therapeutics (27). The frequency of gene alterations in BTC is comparatively high, particularly so in IDH as well as in the TP53, KRAS, and
FGFR pathways (28). Additionally, the three subtypes of BTC have substantial molecular heterogeneity (29). The FGFR fusions, TP53, KRAS, IDH1/2, and BAP1 mutations, are the most common mutation in iCCA, while PRKACA, PRKACB fusions, and ELF3 mutations, frequently occur in p/dCCA $(29,30)$. Lossof-function mutations of tumor suppressor genes have been associated with a worse prognosis in BTC. Interestingly, Apurva Jain and colleagues found that FGFR genetic aberrations may have a positive impact on OS in younger patients with an indolent disease course (31). Identification of distinct patient subgroups is associated with targeted therapies. Indeed, gene sequencing leads the way for precision medicine in BTC (32). Besides, another study found BRCA-associated protein 1(BAP1) mutation in CCA may be associated with aggressive disease and poor response to standard therapies (33). 
This study had several limitations. Firstly, the study evaluated the anti-tumor activity of lenvatinib as a non-first-line therapy in patients with advanced BTC using a single-arm design and with a limited number of patients, which limits the strength of evidence and lacks a comparator. Secondly, patients enrolled are from diverse regions of China, which may lead to the bias of the heterogeneity caused by geography. In addition, this study is an investigator-initiated study with underlying selection bias and participant bias. Finally, the mPFS in this study was lower compared to FOLFOX chemotherapy.

In conclusion, the results of this study suggest that lenvatinib which was individualized based on the patient's weight has promising clinical activity against advanced BTC. Besides, AEs caused by levatinib appear to be closely related to the administered dose, and timely detection and treatment of AEs can reduce the occurrence of some AEs. Changes in tumor biomarkers before and after administration of lenvatinib can potentially be used to predict changes in the size of tumors during therapy, with a sensitivity and specificity of approximately $70 \%$. Gene detection is likely to provide guideposts for precision medicine as we move forward in identifying effective therapies for BTC.

\section{DATA AVAILABILITY STATEMENT}

The original contributions presented in the study are included in the article/supplementary material. Further inquiries can be directed to the corresponding authors.

\section{REFERENCES}

1. Bridgewater JA, Goodman KA, Kalyan A, Mulcahy MF. Biliary Tract Cancer: Epidemiology, Radiotherapy, and Molecular Profiling. Am Soc Clin Oncol Educ Book (2016) 35:e194-203. doi: 10.1200/edbk_160831

2. Xue R, Li R, Wang J, Tong W, Hao J. Horizons on the Therapy of Biliary Tract Cancers: A State-Of-the-Art Review. J Clin Transl Hepatol (2021) 9(4):55967. doi: $10.14218 /$ jcth.2021.00007

3. Valle J, Wasan H, Palmer DH, Cunningham D, Anthoney A, Maraveyas A, et al. Cisplatin Plus Gemcitabine Versus Gemcitabine for Biliary Tract Cancer. N Engl J Med (2010) 362(14):1273-81. doi: 10.1056/NEJMoa0908721

4. Motoyama H, Kobayashi A, Yokoyama T, Shimizu A, Kitagawa N, Notake T, et al. Survival Benefits of Surgical Resection in Patients With Recurrent Biliary Tract Carcinoma. World J Surg (2017) 41(11):2817-29. doi: 10.1007/s00268017-4107-3

5. Marin JJG, Prete MG, Lamarca A, Tavolari S, Landa-Magdalena A, Brandi G, et al. Current and Novel Therapeutic Opportunities for Systemic Therapy in Biliary Cancer. Br J Cancer (2020) 123(7):1047-59. doi: 10.1038/s41416-0200987-3

6. Lamarca A, Palmer DH, Wasan HS, Ross PJ, Ma YT, Arora A, et al. SecondLine FOLFOX Chemotherapy Versus Active Symptom Control for Advanced Biliary Tract Cancer (ABC-06): A Phase 3, Open-Label, Randomised, Controlled Trial. Lancet Oncol (2021) 22(5):690-701. doi: 10.1016/s14702045(21)00027-9

7. Lamarca A, Barriuso J, McNamara MG, Valle JW. Molecular Targeted Therapies: Ready for "Prime Time" in Biliary Tract Cancer. J Hepatol (2020) 73(1):170-85. doi: 10.1016/j.jhep.2020.03.007

8. Yi JH, Thongprasert S, Lee J, Doval DC, Park SH, Park JO, et al. A Phase II Study of Sunitinib as a Second-Line Treatment in Advanced Biliary Tract

\section{ETHICS STATEMENT}

The studies involving human participants were reviewed and approved by Institutional Review Board and Ethics Committee at Peking Union Medical College Hospital. The patients/ participants provided their written informed consent to participate in this study.

\section{AUTHOR CONTRIBUTIONS}

YCW, DXW, and XBY collected the data and wrote the manuscript. XY, YYW, JYL, JXZ and ZHL helped collect literature and participated in discussions. MG and $\mathrm{HZ}$ designed and verified the study. YLM and XTS examined the language. All authors contributed to the article and approved the submitted version.

\section{FUNDING}

This work was supported by the International Science and Technology Cooperation Projects (2016YFE0107100), the Capital Special Research Project for Health Development (20142-4012), the Beijing Natural Science Foundation (L172055 and 7192158), the National Ten-thousand Talent Program, the Fundamental Research Funds for the Central Universities (3332018032), and the CAMS Innovation Fund for Medical Science (CIFMS) (2017-I2M-4-003 and 2018-I2M-3-001).

Carcinoma: A Multicentre, Multinational Study. Eur J Cancer (2012) 48 (2):196-201. doi: 10.1016/j.ejca.2011.11.017

9. Takahashi H, Ojima H, Shimizu H, Furuse J, Furukawa H, Shibata T. Axitinib (AG-013736), an Oral Specific VEGFR TKI, Shows Potential Therapeutic Utility Against Cholangiocarcinoma. Jpn J Clin Oncol (2014) 44(6):570-8. doi: 10.1093/jjco/hyu045

10. Goyal L, Zheng H, Yurgelun MB, Abrams TA, Allen JN, Cleary JM, et al. A Phase 2 and Biomarker Study of Cabozantinib in Patients With Advanced Cholangiocarcinoma. Cancer (2017) 123(11):1979-88. doi: 10.1002/cncr.30571

11. Iyer RV, Pokuri VK, Groman A, Ma WW, Malhotra U, Iancu DM, et al. A Multicenter Phase II Study of Gemcitabine, Capecitabine, and Bevacizumab for Locally Advanced or Metastatic Biliary Tract Cancer. Am J Clin Oncol (2018) 41(7):649-55. doi: 10.1097/coc.0000000000000347

12. Krook MA, Lenyo A, Wilberding M, Barker H, Dantuono M, Bailey KM, et al. Efficacy of FGFR Inhibitors and Combination Therapies for Acquired Resistance in FGFR2-Fusion Cholangiocarcinoma. Mol Cancer Ther (2020) 19(3):847-57. doi: 10.1158/1535-7163.Mct-19-0631

13. Kudo M, Finn RS, Qin S, Han KH, Ikeda K, Piscaglia F, et al. Lenvatinib Versus Sorafenib in First-Line Treatment of Patients With Unresectable Hepatocellular Carcinoma: A Randomised Phase 3 Non-Inferiority Trial. Lancet (2018) 391(10126):1163-73. doi: 10.1016/s0140-6736(18)30207-1

14. Arora S, Balasubramaniam S, Zhang W, Zhang L, Sridhara R, Spillman D, et al. FDA Approval Summary: Pembrolizumab Plus Lenvatinib for Endometrial Carcinoma, a Collaborative International Review Under Project Orbis. Clin Cancer Res (2020) 26(19):5062-7. doi: 10.1158/10780432.Ccr-19-3979

15. Vergote I, Powell MA, Teneriello MG, Miller DS, Garcia AA, Mikheeva ON, et al. Second-Line Lenvatinib in Patients With Recurrent Endometrial Cancer. Gynecol Oncol (2020) 156(3):575-82. doi: 10.1016/j.ygyno.2019.12.039 
16. Taylor MH, Lee CH, Makker V, Rasco D, Dutcus CE, Wu J, et al. Phase IB/II Trial of Lenvatinib Plus Pembrolizumab in Patients With Advanced Renal Cell Carcinoma, Endometrial Cancer, and Other Selected Advanced Solid Tumors. J Clin Oncol (2020) 38(11):1154-63. doi: 10.1200/jco.19.01598

17. Lin J, Yang X, Long J, Zhao S, Mao J, Wang D, et al. Pembrolizumab Combined With Lenvatinib as Non-First-Line Therapy in Patients With Refractory Biliary Tract Carcinoma. Hepatobiliary Surg Nutr (2020) 9 (4):414-24. doi: 10.21037/hbsn-20-338

18. El-Khoueiry AB, Rankin CJ, Ben-Josef E, Lenz HJ, Gold PJ, Hamilton RD, et al. SWOG 0514: A Phase II Study of Sorafenib in Patients With Unresectable or Metastatic Gallbladder Carcinoma and Cholangiocarcinoma. Invest New Drugs (2012) 30(4):1646-51. doi: 10.1007/s10637-011-9719-0

19. Sun W, Patel A, Normolle D, Patel K, Ohr J, Lee JJ, et al. A Phase 2 Trial of Regorafenib as a Single Agent in Patients With Chemotherapy-Refractory, Advanced, and Metastatic Biliary Tract Adenocarcinoma. Cancer (2019) 125 (6):902-9. doi: 10.1002/cncr.31872

20. Javle M, Lowery M, Shroff RT, Weiss KH, Springfeld C, Borad MJ, et al. Phase II Study of BGJ398 in Patients With FGFR-Altered Advanced Cholangiocarcinoma. J Clin Oncol (2018) 36(3):276-82. doi: 10.1200/jco.2017.75.5009

21. Abou-Alfa GK, Macarulla T, Javle MM, Kelley RK, Lubner SJ, Adeva J, et al. Ivosidenib in IDH1-Mutant, Chemotherapy-Refractory Cholangiocarcinoma (ClarIDHy): A Multicentre, Randomised, Double-Blind, Placebo-Controlled, Phase 3 Study. Lancet Oncol (2020) 21(6):796-807. doi: 10.1016/s1470-2045 (20)30157-1

22. Ueno M, Ikeda M, Sasaki T, Nagashima F, Mizuno N, Shimizu S, et al. Phase 2 Study of Lenvatinib Monotherapy as Second-Line Treatment in Unresectable Biliary Tract Cancer: Primary Analysis Results. BMC Cancer (2020) 20 (1):1105. doi: 10.1186/s12885-020-07365-4

23. Zhao Y, Zhang YN, Wang KT, Chen L. Lenvatinib for Hepatocellular Carcinoma: From Preclinical Mechanisms to Anti-Cancer Therapy. Biochim Biophys Acta Rev Cancer (2020) 1874(1):188391. doi: 10.1016/j.bbcan.2020.188391

24. Torrens L, Montironi C, Puigvehí M, Mesropian A, Leslie J, Haber PK, et al. Immunomodulatory Effects of Lenvatinib Plus Anti-Programmed Cell Death Protein 1 in Mice and Rationale for Patient Enrichment in Hepatocellular Carcinoma. Hepatology (2021) 74(5):2652-69. doi: 10.1002/hep.32023

25. Hegde PS, Wallin JJ, Mancao C. Predictive Markers of Anti-VEGF and Emerging Role of Angiogenesis Inhibitors as Immunotherapeutics. Semin Cancer Biol (2018) 52(Pt 2):117-24. doi: 10.1016/j.semcancer.2017.12.002

26. Valle JW, Kelley RK, Nervi B, Oh DY, Zhu AX. Biliary Tract Cancer. Lancet (2021) 397(10272):428-44. doi: 10.1016/s0140-6736(21)00153-7

27. Jusakul A, Cutcutache I, Yong CH, Lim JQ, Huang MN, Padmanabhan N, et al. Whole-Genome and Epigenomic Landscapes of Etiologically Distinct
Subtypes of Cholangiocarcinoma. Cancer Discov (2017) 7(10):1116-35. doi: 10.1158/2159-8290.Cd-17-0368

28. Churi CR, Shroff R, Wang Y, Rashid A, Kang HC, Weatherly J, et al. Mutation Profiling in Cholangiocarcinoma: Prognostic and Therapeutic Implications. PloS One (2014) 9(12):e115383. doi: 10.1371/journal.pone.0115383

29. Banales JM, Marin JJG, Lamarca A, Rodrigues PM, Khan SA, Roberts LR, et al. Cholangiocarcinoma 2020: The Next Horizon in Mechanisms and Management. Nat Rev Gastroenterol Hepatol (2020) 17(9):557-88. doi: 10.1038/s41575-020-0310-Z

30. Nakamura H, Arai Y, Totoki Y, Shirota T, Elzawahry A, Kato M, et al. Genomic Spectra of Biliary Tract Cancer. Nat Genet (2015) 47(9):1003-10. doi: 10.1038/ng.3375

31. Jain A, Borad MJ, Kelley RK, Wang Y, Abdel-Wahab R, Meric-Bernstam F, et al. Cholangiocarcinoma With FGFR Genetic Aberrations: A Unique Clinical Phenotype. JCO Precis Oncol (2018) 2:1-12. doi: 10.1200/ po. 17.00080

32. Rizvi S, Gores GJ. Emerging Molecular Therapeutic Targets for Cholangiocarcinoma. J Hepatol (2017) 67(3):632-44. doi: 10.1016/ j.jhep.2017.03.026

33. Al-Shamsi HO, Anand D, Shroff RT, Jain A, Zuo M, Conrad C, et al. BRCAAssociated Protein 1 Mutant Cholangiocarcinoma: An Aggressive Disease Subtype. J Gastrointest Oncol (2016) 7(4):556-61. doi: 10.21037/ jgo.2016.03.05

Conflict of Interest: The authors declare that the research was conducted in the absence of any commercial or financial relationships that could be construed as a potential conflict of interest.

Publisher's Note: All claims expressed in this article are solely those of the authors and do not necessarily represent those of their affiliated organizations, or those of the publisher, the editors and the reviewers. Any product that may be evaluated in this article, or claim that may be made by its manufacturer, is not guaranteed or endorsed by the publisher.

Copyright (c) 2022 Wang, Yang, Wang, Yang, Wang, Long, Zhou, Lu, Mao, Sang, Guan and Zhao. This is an open-access article distributed under the terms of the Creative Commons Attribution License (CC BY). The use, distribution or reproduction in other forums is permitted, provided the original author(s) and the copyright owner(s) are credited and that the original publication in this journal is cited, in accordance with accepted academic practice. No use, distribution or reproduction is permitted which does not comply with these terms. 